\title{
INFLUÊNCIA DO ÍNDICE DE MASSA CORPORAL NO EQUILÍBRIO DE IDOSAS ATIVAS PARTICIPANTES DE PROJETO UNATI DE ATIVIDADE FÍSICA
}

Jaqueline Luche Neves, Bruna Moreira Garcia, Elaine Aparecida Lozano da Silva, Ana Caroline Rippi Moreno, Regina Celi Trindade Camargo.

Universidade Estadual Paulista Julio de Mesquita Filho, Curso de Graduação em Fisioterapia, Presidente Prudente, São Paulo.E-mail: jaqueluche@hotmail.com

\section{RESUMO}

O envelhecimento traz modificações funcionais e a alteração de equilíbrio é uma das principais queixas. A falta de equilíbrio pode levar a quedas e agravos à saúde do idoso. O objetivo do estudo foi avaliar a influência do índice de massa corporal no equilíbrio de idosas ativas. Foi realizada avaliação antropométrica e aplicação do teste de Apoio Unipodal em participantes de programa fisioterapêutico regular de exercícios físicos. Os resultados obtidos foram idade média de 71,16 anos, IMC total de $27,38 \mathrm{Kg} / \mathrm{m}^{2}$ e Mini Exame de Estado Mental (MEEM) 25,9 $\pm 3,3$ pontos, sendo média do teste Unipodal com olhos abertos foi de 30 segundos para G. Abaixo, 22,75 segundos para G. Normal e 21,08 segundos para G. Acima. Para o teste Unipodal com olhos fechados o G. Normal obteve 7,15 segundos, G. Abaixo 4,75 segundos G. Acima 4,43 segundos. Os resultados demonstraram significância estatística no equilíbrio dos participantes que possuíam IMC ideal, seguido de IMC abaixo.

Palavras-chave: idoso, equilíbrio, atividade física, envelhecimento, saúde.

\section{MASS INDEX OF INFLUENCE BODY IN OLDER LIVE PROJECT PARTICIPANTS BALANCE PHYSICAL ACTIVITY UNATI}

\begin{abstract}
Aging brings functional changes and the balance change is one of the main complaints. The lack of balance can lead to falls and health problems of the elderly. The aim of the study was to evaluate the influence of body mass index in the balance of active elderly women. Anthropometric and application of unipodal test participants in regular physical therapy exercise program evaluation was carried out. The results were mean age 71.16 years old, BMI Total $27,38 \mathrm{Kg} / \mathrm{m}^{2}$ and mini-mental state examination (MMSE) $25.9 \pm 3.3$ points and average unipodal test with open eyes was 30 seconds to G. Below 22.75, seconds to G. Normal and 21.08 seconds to $G$. Above. For the one-leg test with eyes closed G. Normal obtained 7.15 seconds, G. Below 4,75 seconds and $G$. Above 4.43 seconds. The results showed statistical significance in the balance of participants had ideal BMI, followed by BMI below.
\end{abstract}

Keywords: elderly, balance, physical activity, aging, health.

\section{INTRODUÇÃO}

Atualmente $\quad 0$ envelhecimento populacional é um fenômeno global, sendo o número progressivo de idosos um desafio para a sociedade, pois, o aumento da idade vem seguido de modificações funcionais desfavoráveis ao idoso, que consequentemente afetam na sua qualidade de vida. Essas modificações alteram a capacidade funcional do ser humano, com o passar dos anos podendo levar à incapacidade funcional'.

O declínio da capacidade funcional compromete diversos sistemas, dentre eles 0 sistema nervoso central e periférico, estruturas ósseas, músculos e articulações, estes por sua vez quando modificados prejudicam o processamento dos sinais responsáveis pelo equilíbrio corporal ${ }^{2}$.

As alterações do equilíbrio são uma das queixas mais comuns encontradas na população idosa, e podem se manifestar por meio de desvios da marcha, instabilidades e náuseas, estes associados ao maior risco de quedas e consequentes agravos à saúde ${ }^{3}$.

A queda é a consequência da perda total do equilíbrio postural e dos mecanismos 
envolvidos na manutenção da postura, pode ser definida como a mudança inesperada e não intencional de posição do indivíduo para um nível mais baixo, em relação à posição inicial, esta tem se manifestado como um dos principais problemas de saúde pública devido às implicações que ocasionam ${ }^{4}$.

A queda no idoso pode ser favorecida tanto por fatores intrínsecos, quanto extrínsecos. Os fatores extrínsecos estão associados aos desafios do ambiente em que vivem, e os fatores intrínsecos estão relacionados às alterações fisiológicas do envelhecimento, as doenças e efeitos de medicamentos ${ }^{5}$.

Após a ocorrência de quedas, os idosos apresentam dificuldades em realizar as atividades de vida diária, por desenvolvimento de complicações, como o declínio funcional do organismo, quadros álgicos, incapacidades e hospitalizações. A ocorrência de fraturas é uma das consequências mais comuns após as quedas, sendo a fratura do fêmur a mais frequente e considerada a mais importante em termos de morbidade, mortalidade e custos, pois está associada ao maior tempo de internação e cuidados ${ }^{6}$.

Desse modo tornam-se necessárias estratégias que atuam na prevenção do surgimento de quedas, tais como a prática de atividade física, pois proporciona estímulos ao equilíbrio, aprimorando recepções de informações sensoriais do sistema vestibular, visual e somatossensorial ${ }^{7}$. Também a atividade física proporciona o aumento da massa muscular, melhora do condicionamento aeróbico, flexibilidade e equilíbrio, dessa forma atua na melhora na qualidade de vida e redução do risco de quedas ${ }^{8}$.

Outro fator que interfere no equilíbrio de idosos é o valor elevado do Índice de Massa Corpórea (IMC). O IMC esteve associado a um pior desempenho nos testes de Apoio Unipodal e Velocidade Máxima ao Andar, em estudos que utilizaram como ferramenta metodológica o teste de Apoio Unipodal, e o teste de Velocidade Máxima ao Andar ${ }^{9,10}$.

Diante do apresentado, o estudo teve como objetivo avaliar a influência do índice de massa corporal no equilíbrio de idosas ativas participantes da Universidade Aberta da Terceira Idade (UNATI).

\section{METODOLOGIA}

O estudo com característica longitudinal, aprovado pelo Comitê de Ética e Pesquisa da Unesp de Presidente Prudente, (CAAE no 56496816.3.0000.5402), foi realizado com idosas ativas inseridas no programa de fisioterapia preventiva em geriatria e gerontologia da UNATI de Presidente Prudente, vinculada ao Setor de Gerontologia do Departamento de Fisioterapia da FCT/UNESP.

um protocolo de exercícios físicos supervisionados, composto de alongamento, aquecimento, exercícios para fortalecimento muscular, flexibilidade, coordenação, equilíbrio, memória, dinâmica e relaxamento, com a realização três sessões semanais, com uma hora de duração cada.

Todos os indivíduos foram comunicados quanto ao objetivo do estudo, e após aceitação e assinatura do termo de consentimento livre e esclarecido (TCLE), participaram ativamente da pesquisa.

\section{Critérios de Inclusão:}

Indivíduos do sexo feminino, com idade igual ou superior a 60 anos; frequentar o setor de Fisioterapia em Gerontologia da FCT/UNESP a pelo menos seis meses; Estabilidade clínica e atestado médico, constatando que o mesmo está apto a realizar exercícios físicos moderados; ausência de diagnóstico médico de enfermidades que interferissem na prática de exercícios físicos (por exemplo, doenças cérebro-vasculares, cardíacas, pneumológicas, ortopédicas, reumáticas); aceitação à proposta de avaliação; assinatura do TCLE.

\section{Procedimentos:}

Inicialmente foi realizada a coleta de idade, peso e altura para calculo do IMC. Após houve a aplicação do teste de apoio Unipodal para avaliar o equilíbrio das participantes. $O$ teste de apoio unipodal é realizado com o membro inferior dominante, onde o indivíduo se equilibra com os olhos abertos e depois com olhos fechados por no máximo 30 segundos. 0 teste é realizado em 3 tentativas, considerando o maior resultado. Quanto maior o tempo de permanência, melhor o escore a favor do equilíbrio. 


\section{Grupos de análise:}

Após a coleta do IMC, para analisar a influência do mesmo com o equilíbrio, as participantes foram subdividas em 3 grupos. De acordo com Cervi et al. ${ }^{11}$ a classificação do IMC para o idoso deve ser de abaixo do peso quando resultado menor a $22 \mathrm{Kg} / \mathrm{m}^{2}$ (Grupo Abaixo), Eutrofia / Normal quando maior igual a $22 \mathrm{~kg} / \mathrm{m}^{2}$ e menor igual a $27 \mathrm{~kg} / \mathrm{m}^{2}$ (Grupo Normal) e acima do peso quando maior a $27 \mathrm{~kg} / \mathrm{m}^{2}$ (Grupo Acima).

A análise estatística foi realizada utilizando o software PRISMA, apresentando média e desvio padrão para caracterização das participantes. Após a subdivisão dos 3 grupos (G.Abaixo, G.Normal e G.Acima), foi realizado a análise ANOVA - One Way - Tukey, considerado significante quando $p<0,05$ ).

\section{RESULTADOS}

O presente estudo contou com a participação de 46 idosas, com sua caracterização expressa na tabela 1 . Vale ressaltar que não houve nenhuma exclusão segundo o critério do teste Mini Exame de Estado Mental (MEEM), obtendo média de $25,90 \pm 3,3$ pontos.

Tabela 1. Caracterização das participantes.

\begin{tabular}{cc}
\hline Variáveis & Geral \\
\hline $\mathrm{n}$ & 46 \\
Idade (anos) & $71,3 \pm 8,1$ \\
Peso $(\mathrm{Kg})$ & $63,8 \pm 12,1$ \\
Altura $(\mathrm{m})$ & $1,53 \pm 0,05$ \\
$\mathrm{IMC}\left(\mathrm{Kg} / \mathrm{m}^{2}\right)$ & $27,0 \pm 4,4$ \\
\hline
\end{tabular}

Na tabela 2 observa-se o número de participantes (n), média, desvio padrão e valor de p segundo análise estatística, após a subdivisão das participantes em 3 grupos: Grupo Abaixo do peso (G. Abaixo), Grupo com peso normal (G. Normal) e Grupo Acima do peso (G. Acima).

Apesar de não apresentar diferença estatística significante, é interessante observar nas Figura 1 e 2, que o grupo com maior déficit em relação ao equilíbrio foi o Grupo Acima, visto que este obteve o menor resultado em ambas análises.

Tabela 2. Desempenhos no teste de Apoio Unipodal após subdivisão.

\begin{tabular}{lcccc}
\hline \multicolumn{1}{c}{ Variáveis } & G. Abaixo & G. Normal & G. Acima & Valor de $p$ \\
\hline $\mathrm{n}$ & 4 & 20 & 22 & - \\
$\mathrm{IMC}$ & $19,54 \pm 0,88$ & $24,20 \pm 1,27$ & $31,08 \pm 2,28$ & $0,001^{*}$ \\
Unipodal olhos fechados & $4,8 \pm 2,5$ & $7,2 \pm 5,6$ & $4,5 \pm 2,9$ & 0,2663 \\
Unipodal olhos abertos & $30 \pm 0$ & $22,8 \pm 10,1$ & $21,3 \pm 9,6$ & 0,1392
\end{tabular}

*significante G. Abaixo e G. Normal vs G. Acima. Não há diferença significante na análise do equilíbrio em relação ao teste Unipodal olhos abertos ou fechados.

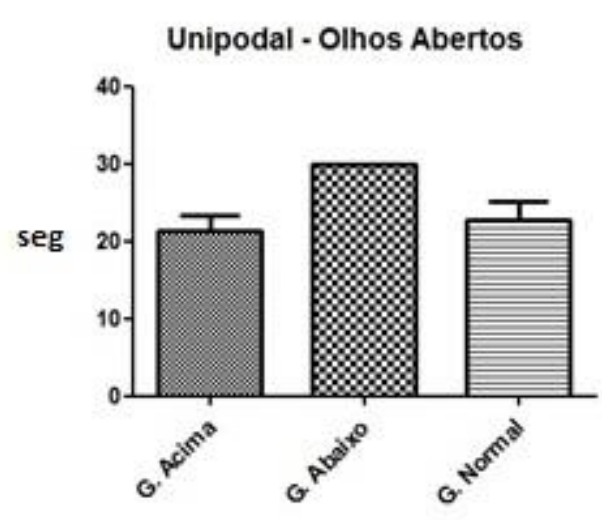

Figura 1. Unipodal - Olhos abertos. Tempo de equilíbrio em segundos. Não houve diferença estatística significante.
Observa-se que o G. Abaixo apresentou resultado maior a favor do equilíbrio no Teste Unipodal com os Olhos Abertos. Em valores porcentuais esse aumento foi de $30 \%$ vs G. Acima e $25 \%$ vs G. Normal. Vale ainda ressaltar que não houve desvio padrão nesse grupo, sendo que todas participantes atingiram o tempo máximo de 30 segundos.

Observa-se que o G. Normal apresentou resultado maior a favor do equilíbrio no Teste Unipodal com os Olhos Fechados. Em valores porcentuais esse aumento foi de $37 \%$ vs G. Acima e $33 \%$ vs G. Abaixo. No entanto, vale ressaltar que o maior desvio padrão foi nesse grupo, sendo de 5,6 segundos. 


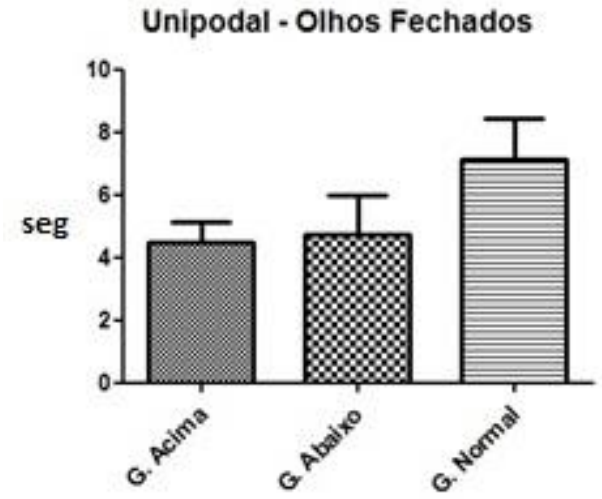

Figura 2. Unipodal - Olhos fechados. Tempo de equilíbrio em segundos. Não houve diferença estatística significante.

\section{DISCUSSÃO}

Os achados do presente estudo demonstraram que há influencia do IMC nos resultados quanto à relação de equilíbrio do idoso. O Teste Unipodal com os olhos fechados nos mostra que o subgrupo de IMC ideal (G. Normal) apresentou uma média de 2,4 segundos a mais de permanência em comparação com o subgrupo G. Abaixo e 2,7 segundos ao G. Acima, ou seja, quanto mais adequado o peso corpóreo, maior o tempo de permanência em equilíbrio ortostático.

No Teste Unipodal com os olhos abertos o subgrupo G. Abaixo conseguiu atingir o tempo máximo de permanência, entretanto o G. Normal teve em média 7,25 segundos a menos, seguido do G. Acima com 8,73 segundos abaixo do tempo máximo estimado.

Com o processo de envelhecimento ocorre naturalmente uma diminuição da força muscular, e se acentua com o aumento de peso, consequentemente, ocorrem impactos negativos na manutenção do equilíbrio devido à perda de massa muscular ${ }^{11}$.

De modo geral, a obesidade pode comprometer alguns aspectos da função em idosas e está relacionada com fatores de instabilidade, diminuição da qualidade de vida e limitações funcionais ${ }^{12,13}$. Porém, contrariando estes resultados, Era et al. $^{14}$, observaram associação entre o alto IMC e o bom desempenho nos testes de equilíbrio estático e dinâmico.

Foi possível observar que as participantes com IMC elevado, ou seja, as que apresentaram acima de $27 \mathrm{~kg} / \mathrm{m}^{2}$ obtiveram, em média, resultado inferior para o Teste Unipodal com os olhos abertos e fechados em relação aos que apresentaram IMC normal ou abaixo.

A obesidade é um fator associado ao risco de quedas em idosos, tendo impacto negativo no equilíbrio e oscilação postural, além de aumentar o risco de limitações funcionais. Quando comparado o equilíbrio de jovens obesos e não-obesos, Goulding et al. ${ }^{15}$, não encontraram diferenças no equilíbrio com base de sustentação bipodal, em contrapartida, no equilíbrio unipodal houve alteração significativa, acredita-se que pela redução da base de suporte, o que corrobora com Greve et al. ${ }^{16}$, observaram correlação entre altos valores de IMC e instabilidade postural em suporte unipodal.

Entende-se que nas diferentes faixas etárias, a quantidade de gordura corporal e a maior dimensão do corpo podem dificultar o posicionamento das pernas e a manutenção do equilíbrio no Teste Unipodal ${ }^{16}$.

Barbosa et al. ${ }^{17}$, evidenciam que mulheres obesas a partir da meia idade começam a apresentam maior déficit de equilíbrio estático quando comparadas às não obesas, sugerindo que, embora o envelhecimento acarrete degeneração dos sistemas de controle postural, a obesidade é um fator agravante.

\section{CONCLUSÃO}

Desse modo pode-se concluir que altos valores de IMC podem interferir de forma negativa no equilíbrio de idosas ativas, contribuindo para um pior desempenho tanto com os olhos abertos, quanto com os olhos fechados.

\section{CONFLITO DE INTERESSE}

Os autores declaram não haver qualquer potencial de conflito de interesse que possa interferir na imparcialidade deste trabalho científico.

\section{REFERÊNCIAS}

1. Cunha MF, Lazzareschi L, Gantus MC, Suman MR, Silva $A$, Parizi CC et al. A influência da fisioterapia na prevenção de quedas em idosos na comunidade: estudo comparativo. Motriz. 2009;15(3):527-36.

2. Moura MS, Pedrosa MAC, Costa EL, Bastos Filho OS, Sayão LB, Sousa TS. Efeitos de exercícios resistidos, de equilíbrio sobre a mobilidade funcional de idosas com baixa massa óssea. Rev Bras Ativ Física Saúde. 2012;17(6):474-81. DOI: http://dx.doi.org/10.12820/rbafs.v.17n6p474-484 
3. Simoceli L, Bittar RMS, Bottino MA, Bento RF. Perfil diagnóstico do idoso portador de desequilíbrio corporal: resultados preliminares. Rev Bras Otorrinolaringol. 2003;69(6):772-7. DOI: http://dx.doi.org/10.1590/S003472992003000600008

4. Cavalcante DPM, Silva LJ, Matos N, Borges I, Araújo DP, Pinheiro HA. Perfil e ambiente de idosos, que sofreram quedas, atendidos em um ambulatório de Geriatria e Gerontologia no Distrito Federal. Rev Kairós Gerontol. 2015;18(1):93-107.

5. Santos RKM, Maciel ACC, Britto HMJS, Lima JCC, Souza TO. Prevalência e fatores associados ao risco de quedas em idosos adscritos a uma Unidade Básica de Saúde do município de Natal, RN, Brasil. Ciê Saúde Colet. 2015;20(12):3753-62. DOI: http://dx.doi.org/10.1590/1413-

\subsection{5}

6. Soares DS, Mello LM, Silva AS, Nunes AA. Análise dos fatores associados à queda com fratura de fêmur em idosos: um estudo caso- controle. Rev Bras Geriat Gerontol. 2015;18(2):239-48.

DOI: http://dx.doi.org/10.1590/1809-9823.2015.14022

7. Alves AHC, Patrício ACFA, Albuquerque KF, Duarte MCS, Santos JS, Oliveira MS. Ocorrência de quedas entre idosos institucionalizados: prevalência, causas e consequências. Rev Online Pesq Cuidado fundam. 2016;8(2):4376-86. DOI: http://dx.doi.org/10.9789/2175-5361.2016.v8i2.43674386

8. Marques H, Almeida ACC, Silva DGG, Lima LS, Oliveira ML, Magalhães AT et al. Escala de equilíbrio de Berg: instrumentalização para avaliar qualidade de vida de idosos. Salusvitta, 2016;35(1):53-65.

9. Apovian CM, Frey CM, Wood GC, Rogers JZ, Still CD, Jensen GL. Body mass index and physical function in older women. Obes Res. 2002;10(8):740-7. DOI: http://dx.doi.org/10.1038/oby.2002.101

10. Stenholm S, Rantanen T, Alanen E, Reunanen A, Sainio $P$, Koskinen $S$. Obesity history as a predictor of walking limitation at old age. Obesity. 2007;15(4):92938. DOI: http://dx.doi.org/10.1038/oby.2007.583

11. Cervi A, Franceschini SCC, Priore SE. Análise critica do uso do índice de massa corporal para idosos. Rev Nutr. 2005;18(6):765-75 DOI: http://dx.doi.org/10.1590/S141552732005000600007

12. Francisco CO, Okada VT, Ricci NA, Benze BG, Rebelatto JR, Duarte ACO. Comparação do equilíbrio corporal de mulheres a partir da meia-idade obesas e não-obesa. Fisioter Pesq. 2009;16(4):323-8. DOI: http://dx.doi.org/10.1590/S1809-

\section{7}

13. Davison KK, Ford ES, Cogswell ME, Dietz WH. Percentage of body fat and body mass index are associated with mobility limitations in people aged 70 and older from NHANES III. J Am Geriatr Soc. 2002;50(11):1802-9.
DOI: $\underline{\text { http://dx.doi.org/10.1046/i.1532- }}$

5415.2002.50508.x

14. Era P, Schroll M, Ytting H, Gause-Nilsson I, Heikkinem E, Steen B. Postural balance and its sensory-motor correlates in 75-year-old men and women: a cross-national comparative study. J Gerontol Biol Med Sci. 1996;51(2):M53-M63.

15. Goulding A, Jones IE, Taylor RW, Piggot JM, Taylor D. Dynamic and static tests of balance and postural sway in boys: effects of previous wrist bone fractures and high adiposity. Gait Posture. 2003; 17(2):136-41. DOI: http://dx.doi.org/10.1016/S0966-6362(02)00161$\underline{3}$

16. Greve J, Alonso A, Bordini ACPG, Camanho GL. Correlation between body mass index and postural balance. Clinics. 2007;62(6):717-20. DOI: http://dx.doi.org/10.1590/S1807$\underline{59322007000600010}$

17. Barbosa AR, Souza JMP, Lebrão ML, Marucci MFN. Estado nutricional e desempenho motor de idosos de São Paulo. Rev Assoc Med Bras. 2007;53(1):75-9. DOI: http://dx.doi.org/10.1590/S010442302007000100024

Recebido para publicação em 19/08/2016 Revisado em 09/09/2016

Aceito em 14/09/2016 